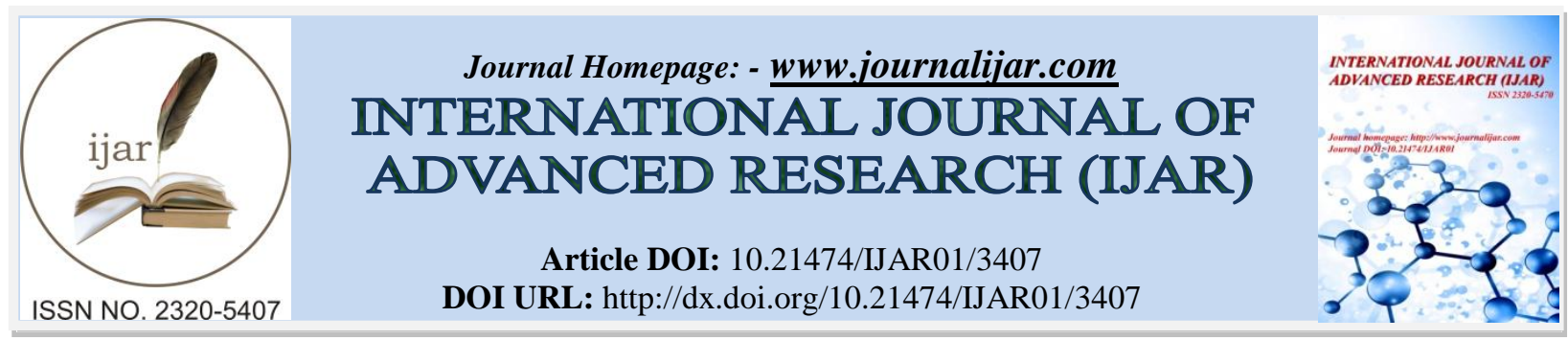

RESEARCH ARTICLE

\title{
DUCTAL CARCINOM A OF BREAST WITH EPITROCHLEAR LYMPH NODE METASTASIS : A RARE CASE REPORT.
}

\author{
Vasudha singh ${ }^{1}$, Vinita Trivedi ${ }^{1}$, Richa Chauhan ${ }^{1}$, Manashi ghosh ${ }^{1}$, Kaustav mandal $^{1}$ and Ravishwar \\ Narayan'. \\ 1. Department of Radiation Oncology, Mahavir Cancer Sansthan. \\ 2. Department of Nuclear Medicine, Mahavir Cancer Sansthan.
}

\section{Manuscript Info}

\section{Manuscript History}

Received: 14 December 2016

Final Accepted: 21 January 2017

Published: February 2017

Key words:-

Carcinoma breast; Invasive ductal

carcinoma; Metastasis; Epitrochlear

lymph node

\begin{abstract}
Metastasis to epitrochlear lymph node from primary invasive breast malignancy is extremely rare and to the best of our knowledge, only one case has been reported in literature till date.

Here we report a case of invasive ductal carcinoma of breast in a premenopausal female who developed metastasis to ipsilateral epitrochlear lymph node almost two and a half years post treatment of the primary tumour.
\end{abstract}

Copy Right, IJAR, 2017,. All rights reserved.

\section{Introduction:-}

Metastatic breast cancer (also known as stage IV or advanced breast cancer) is the cancer that has spread beyond the breast to other organs of the body. The five leading sites of metastatic involvement at autopsy are lung, bone, lymph nodes, liver, and pleura ${ }^{3}$.A few cases of unusual metastasis to urinary bladder, retroperitonium, gall bladder,colon,rectum have been reported before. ${ }^{5,6,7}$ Here we report a post-treated case of breast cancer with metastasis to epitrochlear node, which is an extremely rare site of metastasis. The time interval between treatment of primary tumour and development of metastasis being about two and a half years. The case being presented here, happens to be the second case report of its kind as per literature.

\section{Case Report:-}

A 47 years old premenopausal female presented to our out-patient department in February 2014, with the complaint of painless right breast lump of two months duration. She was a known diabetic and hypertensive and on regular oral medication. On examination, general condition of the patient was good. A $4 \times 4 \mathrm{~cm}$ lump was palpable in the upper outer quadrant of right breast which was non tender, mobile, firm to hard in consistency. Overlying skin and nipple areola complex was normal. There was no palpable lymph node in right axilla and supraclavicular fossa. Contralateral breast ,axilla and supraclavicular fossa was unremarkable. Fine needle aspiration cytology of right breast lump (done outside) was suggestive of ductal carcinoma in situ. Core needle biopsy of the same revealed invasive ductal carcinoma grade II; ER/PR/Her2neu negative. Metastatic work up showed no evidence of metastasis (cT2N0M0). In view of early stage of the disease (stage IIA), upfront modified radical mastectomy was done in April 2014. Post operative histopathology was suggestive of invasive ductal carcinoma grade II; tumour size $3.5 \times 3 \times 3 \mathrm{~cm}$; lymphovascular and perineural invasion negative; lymph nodes 20/20 negative (pT2NOM0). The patient further received six cycles of adjuvant chemotherapy (Cyclophosphamide, Adriamycin, 5 Fluorouracil regimen). She tolerated the treatment well and was kept on regular follow up. However, the patient defaulted. 
In August 2016, patient presented to us again with complaint of painful swelling over the medial aspect of her right elbow of 3 months duration. As per patient, the swelling was rapidly progressive in size. On examination, general condition of the patient was well preserved. Primary disease was locoregionally controlled. Contralateral breast, axilla and supraclavicular fossa was normal. Diffused swelling measuring approximately $10 \mathrm{x} 8 \mathrm{~cm}$ was palpable over medial aspect of right elbow which was tender and inflammed,firm to hard in consistency ,fixed to underlying structures. X ray (right elbow) showed a lobulated soft tissue swelling at posteromedial aspect of lower end of right arm without any evidence of bony involvement. Biopsy of the epitrochlear lymph node showed fibrous tissue infiltrated by tumour composed of malignant epithelial cells having pleomorphic hyperchromatic nuclei exhibiting increased mitosis. Cells arranged in clusters and in glandular pattern in the background of lymphoid tissue, suggestive of metastatic adenocarcinoma; ER/PR/Her2neu negative. Metastatic work up done was unremarkable. As epitrochlear lymph node was the only site of metastasis, patient was adviced for surgical excision with wide margin of the involved lymph node. However she refused any surgical intervention and defaulted to followup.

In January 2017 she again came to us with progression in size of epitrochlear node swelling associated with marked increase in pain along with multiple nodules over right neck region. On examination, general condition of the patient was average, right chest wall and axilla was normal with multiple right supraclavicular lymph nodes, contralteral breast, axilla and supraclavicular fossa was normal. Multiple ulcerated lesions with discharging sinuses were present over medial aspect of right elbow. Multiple non tender, mobile and firm nodules were palpable over right cervical region involving level IV and V; largest measuring $4 \times 3 \mathrm{~cm}$. Fine needle aspiration cytology of the neck node was suggestive of metastatic adenocarcinoma. All other Metastatic work up was otherwise unremarkable. In view of progressive disease patient was further advised for palliative chemotherapy.

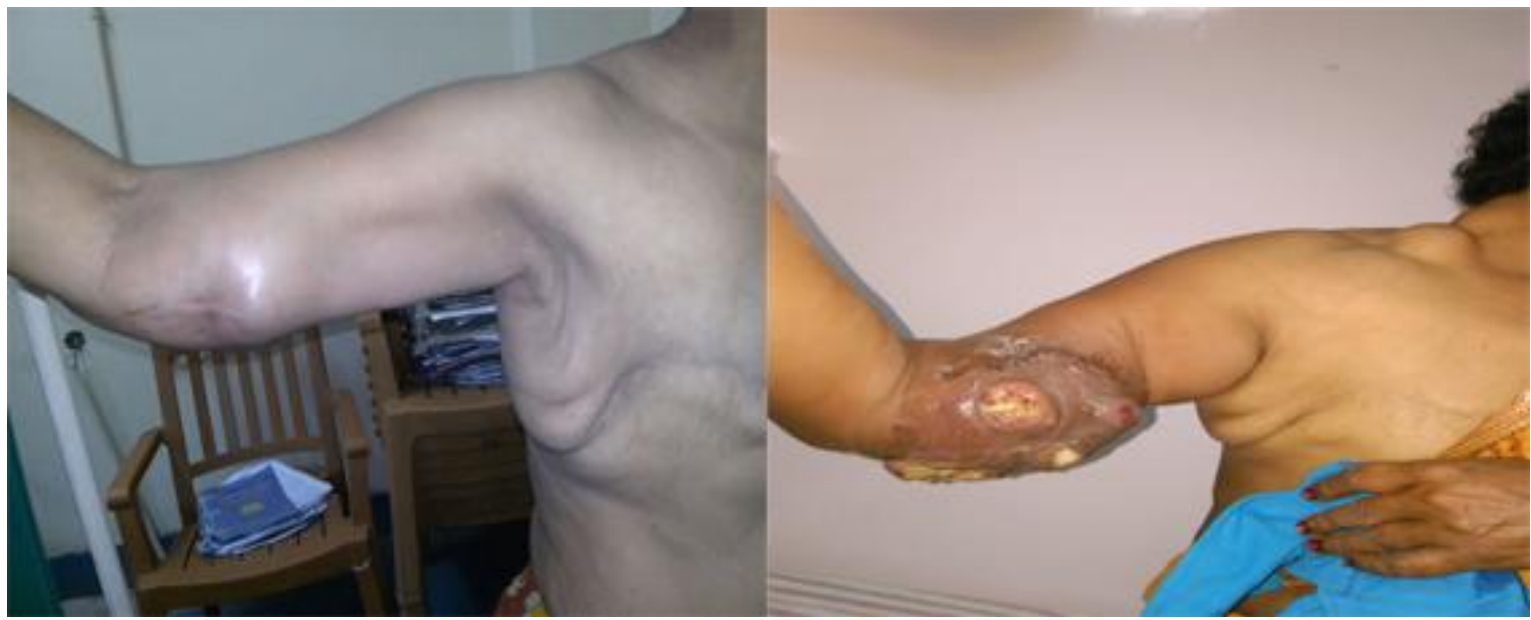

Fig 1:- Metastatic epitrochlear lymph node ,Progressive disease (Epitrochlear with Cervical lymph nodes).

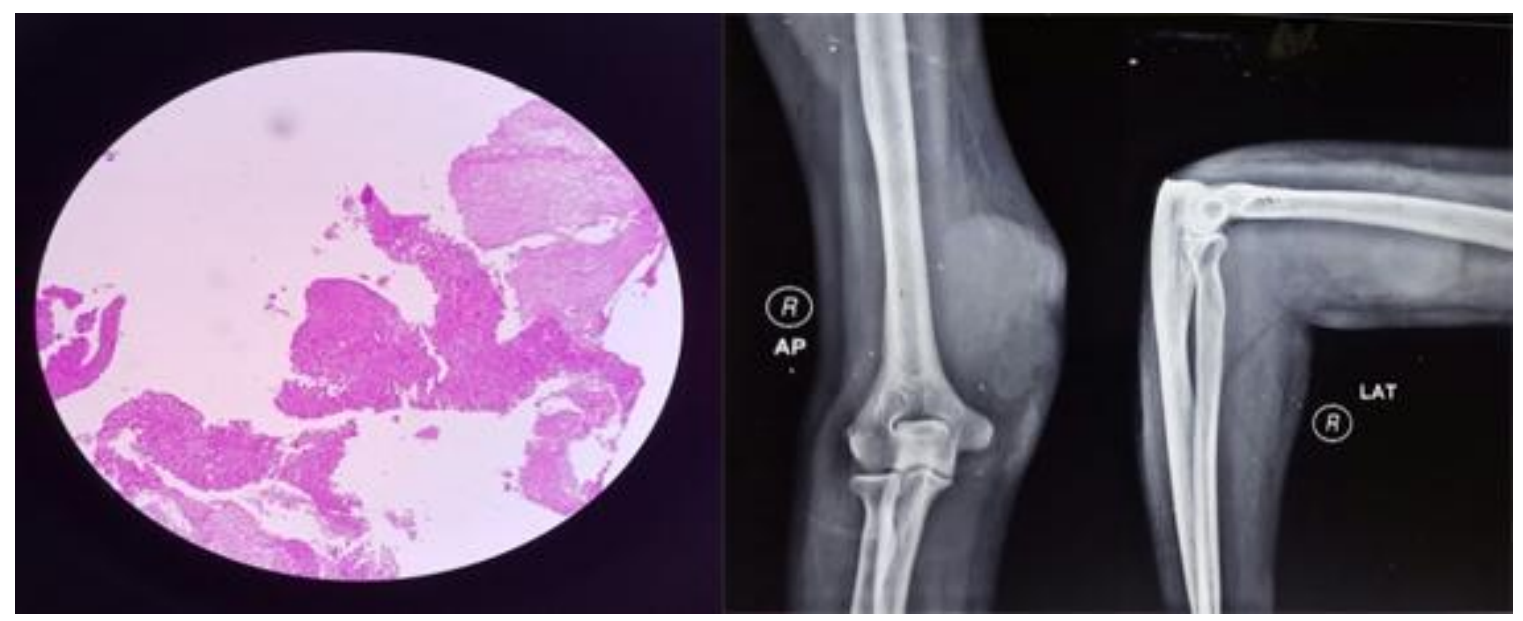

Fig 2:- H\&E of IDC; $X$ ray (Right Elbow). 


\section{Discussion:-}

Breast cancer is the most common malignant disease in females. In these patients, distant metastases is the main cause of death. ${ }^{1}$ Approximately $10-15 \%$ of patients with breast cancer have an aggressive disease and develop distant metastases within 3 years after the initial detection of the primary tumour. ${ }^{2}$ The five leading sites of metastatic involvement at autopsy are lung, bone, lymph nodes, liver, and pleura. The incidence of metastasis in these five sites were: lung 57-77 \%, bone 49-74\%, lymph nodes 50-76\%, liver 50-71\%, and pleura $36-65 \%{ }^{3}$ The most commonly involved axillary lymph nodes are the level I nodes, they are involved in more than $90 \%$ of all patients with any number of positive axillary lymph nodes. Involvement of only level I lymph nodes occur in approximately $60 \%$ of the patients. Involvement of level II lymph nodes without involvement of the level I lymph nodes is uncommon. Involvement of level III lymph nodes with negative levels I and II lymph nodes is rare, occurring in $<3 \%$ of all patients with positive axillary lymph nodes ${ }^{4 . .} \mathrm{A}$ few cases of unusual metastasis to urinary bladder, retroperitonium, gall bladder, colon, rectum have been reported before. ${ }^{5,6,7}$ Lymph node involvement is the most important determinant factor for distant metastasis. In this case all dissected axillary node was negative. The chances of skip metastasis to the epitrochlear lymph node are very rare. The exact pathogenesis is not known for this kind of metastasis. To the best of our knowledge there has been only one case of epitrochlear nodal metastasis reported in literature. ${ }^{8}$

The epitrochlear lymph nodes are part of the superficial lymphatic system of the upper limb. There are usually one or two of these lymph nodes.As a general rule, the epitrochlear station drains the lymph from the last two or three fingers and from the medial aspect of the hand itself. The drainage areas of the upper limb are widely variable interindividually. Some lymphatic vessels arise from the epitrochlear nodes and extend to the axillary station. ${ }^{9}$

Epitrochlear lymph nodes may be enlarged as a part of generalized lymphadenopathy, isolated enlargement of epitrochlear lymph nodes is very rare. Malignant causes include lymphoma and malignant melanoma. In transit metastasis to epitrochlear node are encountered from primary melanoma of the forearm, wrist and hand. Cat scratch disease, leprosy, leishmaniasis and tuberculosis are a few reported benign causes of isolated epitrochlear lymph node enlargement. ${ }^{10}$

There is no definite guideline regarding treatment of epitrochlear nodal metastasis. Surgical resection of the node is to be done where possible, provided there are no other sites of metastasis. This patient was reluctant for surgery, so planned for palliative radiotherapy and was further subjected to chemotherapy on progression of disease.

\section{References:-}

1. Weigelt B, Peterse JL, Van't Veer LJ. Breast cancer metastasis: markers and models. Nature reviews cancer. 2005 Aug 1;5(8):591-602.

2. Hellman, S. \& Harris, J. R. in Diseases of the Breast (eds Harris, J. R., Lippman, M. E., Morrow, M. \& Osborne, C. K.), 407-423 (Lippincott Williams \& Wilkins, Philadelphia, 2000)

3. Lee YT. Breast carcinoma: pattern of metastasis at autopsy. Journal of surgical oncology. 1983 Jul 1;23(3):17580.

4. Sakorafas GH, Tsiotou AG, Balsiger BM. Axillary lymph node dissection in breast cancer: current status and controversies, alternative strategies and future perspectives. ActaOncologica. 2000 Jan 1;39(4):455-66.

5. Shah KG, Modi PR, Rizvi J. Breast carcinoma metastasizing to the urinary bladder and retroperitoneum presenting as acute renal failure. Indian Journal of Urology. 2011 Jan 1;27(1):135.

6. Nemri SN, Haider N, Fatima S, D'sa BF. Unusual sites of metastasis of breast carcinoma to gallbladder and colon.Annals of Pathology and Laboratory Medicine. 2016 Feb 21;3(1):C6-9.

7. Ikeda Y, Morita N, Ikeda T. Metachronous rectal metastasis from invasive ductal carcinoma of the male breast. Endoscopy. 2008 Dec;40(S 02):E108-9.

8. Kumar P, Singh S, Datta NR, Tandon A. Epitrochlear lymph node metastases from invasive ductal breast cancer. Journal of cancer research and therapeutics. 2009 Jul 1;5(3):203.

9. Catalano O, Nunziata A, Saturnino PP, Siani A. Epitrochlear lymph nodes: Anatomy, clinical aspects, and sonography features. Pictorial essay.Journal of ultrasound. 2010 Dec 31;13(4):168-74.

10. Garg PK, Jain BK, Rathi V, Singh N, Mohanty D. Mid-arm and epitrochlear lymphadenopathy: a clinicoradiological surprise. The Journal of Infection in Developing Countries. 2011 Oct 16;5(11):820-4. 